\title{
Optimization and microbial community analysis of anaerobic co-digestion of food waste and sewage sludge based on microwave pretreatment
}

\author{
Junya Zhang $^{\mathrm{a}, \mathrm{b}, 1}$, Chen Lv $^{\mathrm{c}, 1}$, Juan Tong ${ }^{\mathrm{a}, \mathrm{b}}$, Jianwei Liu ${ }^{\mathrm{c}}$, Jibao Liu ${ }^{\mathrm{a}, \mathrm{b}}$, Dawei Yu ${ }^{\mathrm{a}, \mathrm{b}}$, Yawei Wang ${ }^{\mathrm{a}, \mathrm{b}}$, \\ Meixue Chen ${ }^{\mathrm{a}, \mathrm{b}}$, Yuansong Wei ${ }^{\mathrm{a}, \mathrm{b}, *}$

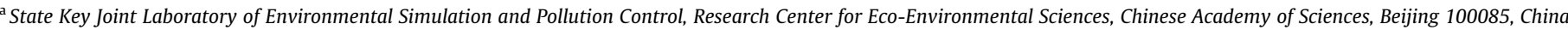 \\ ${ }^{\mathrm{b}}$ University of Chinese Academy of Sciences, Beijing 100049, China \\ ${ }^{\mathrm{c}}$ School of Environment and Energy Engineering, Beijing University of Civil Engineering and Architecture, Beijing 100044, China
}

\section{H I G H L I G H T S}

- Methane production was enhanced by both co-digestion and MW pretreatment.

- The optimized ratio was 3:2 for anaerobic co-digestion based on MW pretreatment.

- Propionic accumulation led to lower methane production of MW-FW compared to MW-SS.

- Bacteroides only dominated in co-digestion, Methanosphaera only dominated in MW-FW.

- Methanosarcina was the predominant methanogens for mono-SS and MW-SS.

\section{A R T I C L E I N F O}

\section{Article history:}

Received 23 August 2015

Received in revised form 12 October 2015

Accepted 13 October 2015

Available online 19 October 2015

\section{Keywords:}

Anaerobic co-digestion

Sewage sludge

Food waste

Bacterial community

Archaeal community

\begin{abstract}
A B S T R A C T
The effects of microwave pretreatment (MW) on co-digestion of food waste (FW) and sewage sludge (SS) have never been investigated. In this study, a series of mesophilic biochemical methane potential (BMP) tests were conducted to determine the optimized ratio of FW and SS based on MW, and the evolution of bacterial and archaeal community was investigated through high-throughput sequencing method. Results showed that the optimized ratio was 3:2 for co-digestion of FW and SS based on MW, and the methane production was 316.24 and $338.44 \mathrm{mLCH}_{4} / \mathrm{gVS}_{\text {added }}$ for MW-FW and MW-SS, respectively. The MW-SS was superior for methane production compared to MW-FW, in which accumulation of propionic acid led to the inhibition of methanogenesis. Proteiniborus and Parabacteroides were responsible for proteins and polysaccharides degradation for all, respectively, while Bacteroides only dominated in codigestion. Methanosphaera dominated in MW-FW at the active methane production phase, while it was Methanosarcina in MW-SS and mono-SS.
\end{abstract}

(c) 2015 Elsevier Ltd. All rights reserved.

\section{Introduction}

Sewage sludge (SS) production had an average annual growth of $13 \%$ from 2007 to 2013 , and 6.25 million tons dry solids were produced in 2013 in China, and almost $80 \%$ of it has not been properly stabilized (Yang et al., 2015). The treatment of sewage sludge has become a big challenge due to strict legal regulations, land

\footnotetext{
* Corresponding author at: State Key Joint Laboratory of Environmental Simulation and Pollution Control, Research Center for Eco-Environmental Sciences, Chinese Academy of Sciences, Beijing 100085, China. Tel./fax: +861062849690. E-mail address: yswei@rcees.ac.cn (Y. Wei).

1 Junya Zhang and Chen Lv contributed equally to this work.
}

shortages, rising costs, and public concern along with fast urbanization and economic development (Wei et al., 2003). Anaerobic digestion (AD) of SS due to the production of renewable energy and sludge reduction has been carried out widely, and ca. $50 \%$ of the total of 2500 waste water treatment plants (WWTPs) were designed with AD up to 2010 in China (Duan et al., 2012). However, its efficiency is largely limited due to the relatively slow hydrolysis process and low $\mathrm{C} / \mathrm{N}$ ratio (6-9), and hydrolysis has been reported as the rate-limiting step (Toreci et al., 2009).

About $6.0 \times 10^{7}$ tons of food waste (FW) were produced according to China Statistical Yearbook 2011, and the increasing rate of FW production was higher than $10 \%$ every year due to population growth and rising living standards. AD is preferred as an efficient 
pathway for treatment of FW due to its high organic contents and excellent biodegradability (Zhang et al., 2007). Nevertheless, the accumulation of volatile fatty acids (VFAs) occurred frequently due to the labile organic fraction which was hydrolyzed too fast, and this may lead to process failure due to the acidification of AD (Kawai et al., 2014; Yong et al., 2015). Thus, the anaerobic codigestion of FW and SS became increasingly popular, with the advantage of adjusting the $\mathrm{C} / \mathrm{N}$ ratio, increasing the methane yields, diluting harmful substances, and also mediating the hydrolysis of FW and SS (Kawai et al., 2014; Lee et al., 2009; Yong et al., 2015).

Cell wall disruption or destruction is considered as an effective way of enhancing AD efficiency of SS which is mainly composed of microbial cells within an extra-cellular polymeric floc matrix (Baier and Schmidheiny, 1997), because hydrolysis was enhanced through SS pretreatment by releasing the intracellular organics that were easily biodegraded. Microwave pretreatment (MW) of SS (MW-SS) was widely investigated to enhance the AD efficiency of SS, and volatile solids (VS) removal, methane production and AD stabilization were improved (Coelho et al., 2011). Meanwhile, the effects of microwave pretreatment of FW (MW-FW) on AD were investigated, while contrary results existed. Marin et al. (2010) indicated that anaerobic biodegradability could be improved by 9\% after MW of model FW. In contrary, Shahriari et al. (2013) recommended that digester staging without MW be employed to maximize methane production in comparison with MW-FW. However, high temperature $\left(175^{\circ} \mathrm{C}\right.$ or $\left.145^{\circ} \mathrm{C}\right)$ was adopted in these studies during MW pretreatment, there is little information on the effects of MW-FW under low temperature $\left(<100^{\circ} \mathrm{C}\right)$ on the AD. Besides, although the effects of MW-SS or MW-FW on AD efficiency have been investigated, there is still little information concerning the underlying microbial mechanisms.

In addition, although several studies focusing on the bacterial or archaeal community of anaerobic co-digestion of FW and SS have been conducted previously under hyper-thermophilic and thermophilic conditions using PCR-DGGE (Lee et al., 2009) or dry AD by high-throughput sequencing method (Cho et al., 2013), the information on bacterial and archaea community of anaerobic co-digestion of FW and SS based on MW is still unclear, and anaerobic co-digestion of SS and FW based on MW has never been investigated till now to our knowledge. Therefore, the aims of this study were as follows: (1) to determine whether MW could enhance anaerobic co-digestion of FW and SS; (2) to find out the optimized ratio of FW and SS based on MW for anaerobic co-digestion; (3) to clarify the evolution of bacterial and archaeal community under optimized ratio using high-throughput sequencing method.

\section{Methods}

\subsection{Pretreatment of substrates and sludge inoculum}

Food waste (FW) was collected daily from the dining hall of Research Center for Eco-environmental Sciences, Chinese Academy of Sciences, Beijing, China, which mainly contains leftovers of cooked foods like meats, fishes, rice, breads, noodles and vegetables, and the hard matters such as bones, toothpicks, chopsticks and napkins were discarded. Grease in FW was full of oils and fats, and led to acidification of AD easily (Zhu et al., 2011), thus, grease was trapped through washing the food waste for 3-4 times, and then FW was homogenized and crushed to particle size of ca. $2 \mathrm{~mm}$ and stored at $4{ }^{\circ} \mathrm{C}$ before use. Dewatered sewage sludge used for co-digestion with FW was collected from Beijing Qinghe WWTP. Due to the low moisture content of dewatered SS, it is hard to mix thoroughly with FW, thus, moisture content was mediated to ca. $90 \%$ by adding water. A laboratory industrial microwave reactor operating at $2,450 \mathrm{MHz}$ and ambient pressure was used for MW pretreatment of FW and SS with a maximum power and temperature of $1000 \mathrm{~W}, 100^{\circ} \mathrm{C}$, respectively. $300-500 \mathrm{~mL}$ raw FW or SS was heated to $100^{\circ} \mathrm{C}$ by the MW reactor operated at $600 \mathrm{~W}$ without additional retention. MW-FW and MW-SS were then cooled down to room temperature before use. Seeding sludge was collected from a mesophilic AD reactor treating SS in Beijing Xiaohongmen WWTP, and sludge after setting down for ca. 23 days was used for inoculum. The characteristics of FW, SS and seeding sludge were shown in Table 1.

\subsection{BMP (biochemical methane potential) tests}

The BMP test, which is considered as the most suitable method for a relatively easy evaluation of the anaerobic digestibility (Nges and Liu, 2009), was used as a tool for evaluating the methane production and biodegradability of the mixtures of FW and SS at different ratios based on MW. In this study, BMP tests were carried out by an Automatic Methane Potential Test System II (Bioprocess Control, Sweden) in which digesters were a series of serum bottles (working volume: $0.4 \mathrm{~L}$ or $1.8 \mathrm{~L}$ ) equipped with plastic caps including agitators and rubber stoppers. The substances used for BMP tests were mixed thoroughly at a ratio according to total solid (TS) shown in Table 2. $0.4 \mathrm{~L}$ or $1.8 \mathrm{~L}$ of the mixture of substances and inoculums at a ratio of 5:1 (TS) were transferred to each bottle, and the final TS was adjusted to about $3.5 \%$ or $7 \%$ as shown in Table 2. After sealing, the headspaces were flushed with nitrogen gas for 3-5 min to remove traces of oxygen. Then the bottles were incubated in a water bath to control temperature at $37 \pm 0.5^{\circ} \mathrm{C}$. Before biogas production yields were automatically measured, $\mathrm{CO}_{2}$ was removed by a gas-washing bottle containing $3 \mathrm{M} \mathrm{NaOH}$ solution. The termination of BMP tests was judged by no obvious accumulation biogas production from bottles (about 35 days). 19 BMP tests were conducted in total in this study, and there was no sampling for thirteen BMP tests conducted in $0.4 \mathrm{~L}$ working volume bottles which was used to find out the optimized ratio of FW and SS based on MW for anaerobic co-digestion. Three 1.8 L working volume bottles in duplicate were mainly used to find out the mechanisms for the optimized ratio of FW and SS based on MW pretreatment, thus, sampling was conducted on days $1,5,12,19$, 26 and 33 for further analysis.

Table 1

The characteristics of raw materials and feed sludge for anaerobic co-digestion.

\begin{tabular}{|c|c|c|c|c|c|}
\hline Characteristics & Food waste & Dewatered sewage sludge & Inoculated sludge & Microwaved food waste & Microwaved sewage sludge \\
\hline Moisture content (\%) & $88.12 \pm 0.54$ & $89.35 \pm 0.07$ & $97.59 \pm 0.05$ & $87.03 \pm 0.65$ & $88.10 \pm 0.05$ \\
\hline Total solids $(\mathrm{g} / \mathrm{L})$ & $136.83 \pm 11.65$ & $125.87 \pm 7.56$ & $24.19 \pm 1.20$ & $139.67 \pm 0.19$ & $135.77 \pm 1.44$ \\
\hline Volatile solids (g/L) & $130.06 \pm 11.51$ & $84.98 \pm 10.88$ & $12.92 \pm 0.58$ & $132.83 \pm 0.40$ & $101.49 \pm 5.45$ \\
\hline $\operatorname{SCOD}(\mathrm{g} / \mathrm{L})$ & $59.35 \pm 20.65$ & $10.88 \pm 1.68$ & $0.59 \pm 0.02$ & $44.20 \pm 10.56$ & $24.58 \pm 8.70$ \\
\hline $\mathrm{NH}_{4}^{+}-\mathrm{N}(\mathrm{mg} / \mathrm{L})$ & $111.05 \pm 0.90$ & $794.13 \pm 135.88$ & $335.2 \pm 14.80$ & $201.6 \pm 15.70$ & $985.0 \pm 45.70$ \\
\hline Proteins (g/L) & $1.46 \pm 0.10$ & $2.33 \pm 0.55$ & - & $2.12 \pm 0.30$ & $4.83 \pm 0.70$ \\
\hline Polysaccharides (g/L) & $40.16 \pm 22.05$ & $1.10 \pm 0.04$ & - & $19.41 \pm 13.70$ & $2.90 \pm 0.30$ \\
\hline
\end{tabular}




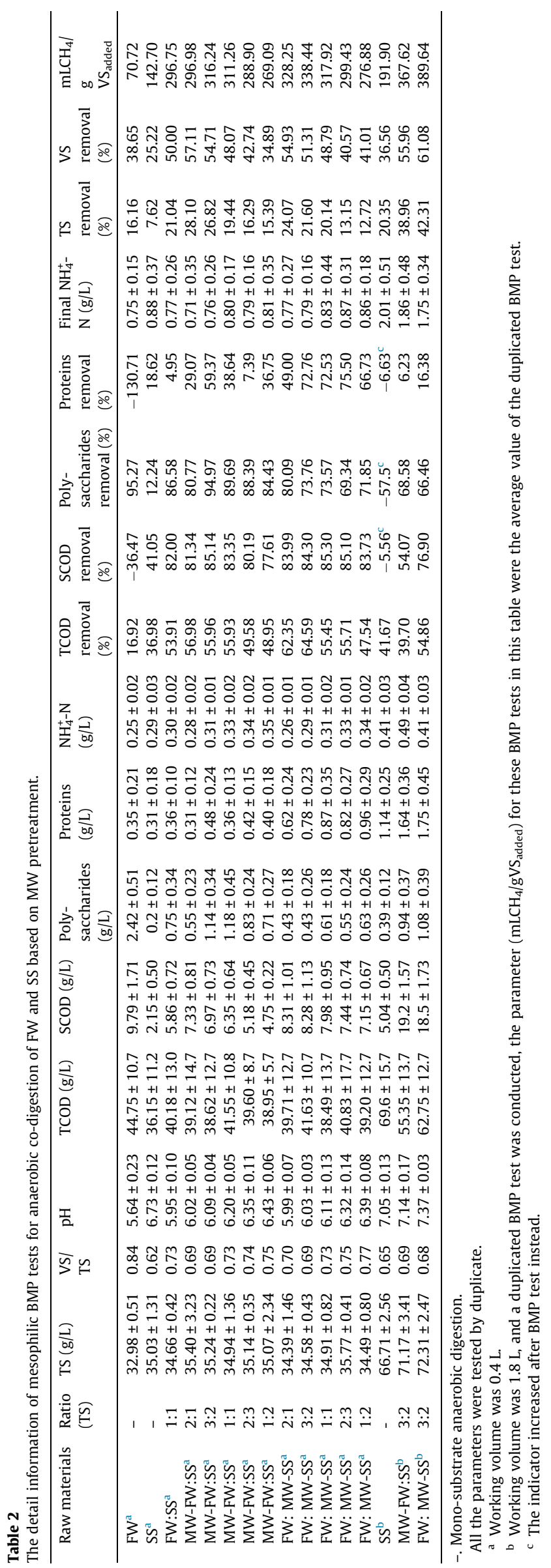

\subsection{Chemical analysis}

TS, TCOD, SCOD, $\mathrm{pH}, \mathrm{NH}_{4}^{+}-\mathrm{N}$ and VS were measured according to standard methods (APHA et al., 2005). Samples were centrifuged at $4000 \mathrm{rpm}$ for $10 \mathrm{~min}$ and filtered through $0.45 \mu \mathrm{m}$ cellulose membrane. The filtrate was analyzed for $\mathrm{pH}, \mathrm{NH}_{4}^{+}-\mathrm{N}, \mathrm{SCOD}$, proteins, polysaccharides, and VFAs. The $\mathrm{pH}$ was determined using a $\mathrm{pH}-$ meter (pHs-3C, Leici Co. Ltd., Shanghai). Ammonia $\left(\mathrm{NH}_{4}^{+}-\mathrm{N}\right)$ was determined by the colorimetric method. SCOD was measured using Hach 8000 methods with a DR 2800 spectrometer (Hach, USA). VFAs were analyzed by a gas chromatography (Shimadzu GC2010 Plus, Japan) equipped with a flame ionization detector (GCFID) and stabilwax-DA column $(0.32 \mathrm{~mm} \times 25 \mathrm{~m} \times 0.25 \mu \mathrm{m})$. The temperatures of the injection port and the detector were set at $240^{\circ} \mathrm{C}$ and $260^{\circ} \mathrm{C}$, respectively, and helium was the carrier gas at the initial pressure of $16.3 \mathrm{kPa}$. The six VFAs standards including acetic acid, propionic acid, butyric acid, iso-butyric acid, valeric acid and iso-valeric acid were purchased from Sigma (Sigma, USA), and the total VFAs concentration in this study was defined as the sum of them. The polysaccharides and proteins content was measured as previously suggested (Zhang et al., 2015).

\subsection{DNA extraction}

Five points of each BMP test for the optimized ratio of FW and SS based on MW on days 1, 5, 12, 19 and 33 were chosen for bacterial and archaeal community analysis. $4 \mathrm{~mL}$ of each sludge sample was centrifuged at $10,000 \mathrm{rpm}$ for $10 \mathrm{~min}$, and the pellet was used for DNA extraction using FASTDNA Spin Kit for Soil (MP Biomedicals, USA) in triplicate according to manufacturer's instructions, and the resulting extracts were composited to average out bias in sampling and extraction. Quality and concentration of the extracted DNA were determined through 1\% agarose gel electrophoresis and NanoDrop ND-1000 (NanoDrop, USA), respectively.

\subsection{Bacterial and archaeal community analysis}

PCR primers $515 \mathrm{~F}$ and $806 \mathrm{R}$ targeting both bacteria and archaea 16S rRNA V4 region were selected for bacterial community analysis using Illumina high-throughput sequencing method (Caporaso et al., 2010). While the abundance of archaeal community was much lower in comparison with bacterial community, archaeal community was specially analyzed using nested PCR. In the nested PCR approach, the specific archaeal community was firstly amplified using the primers Arch340F (5'-CCCTAYGGGGYGCASCAG-3') and Arch1000R (5'-GAGARGWRGTGCATGGCC-3') as described by Gantner et al. (2011), and then the PCR product was used as a template in the second PCR using the primers Arch349F (5'-GYGCAS CAGKCGMGAAW-3') and Arch806R (5'-GGACTACVSGGGTATC TAAT-3') (Takai and Horikoshi, 2000). Barcodes unique to each sample were incorporated before the forward primers, which allowed the identification of each sample in a mixture for an Illumina sequencing run. DNA was amplified in triplicate for each sample, and then PCR amplicons were further purified with a DNA purification kit (Gel Purification kits, Sangon, China), and the concentrations were determined using a spectrometry (Qubit 2.0, Invitrogen, USA). Amplicons from different samples were then mixed to achieve equal mass concentrations in the final mixture, which were sent out to Sangon Co., Ltd., in Shanghai for smallfragment library construction and pair-end sequencing using the Illumina MiSeq sequencing system (Illumina, USA).

Sequencing reads were assigned to each sample according to the unique barcode of each sample. Pairs of reads from the original DNA fragments were firstly merged using FLASH (Magoč and Salzberg, 2011), and then PRINSEQ was used for the quality control 
of these merged reads (Schmieder and Edwards, 2011). The barcode and primers then were removed. All the reads were further uploaded to MG-RAST (http://metagenomics.anl.gov/linkin.cgi? project=14442). PCR chimeras were filtered out using UCHIME (Edgar et al., 2011). After the above filtration, the average length of all of the clean reads was $250 \mathrm{bp}$ and $379 \mathrm{bp}$, and the average sequencing depth was ca. 36,000 and 30,000 clean reads for bacterial and archaeal community analysis, respectively. The taxonomic classification of the sequences was carried out using the Ribosomal Database Project (RDP) Classifier at the bootstrap cutoff of $80 \%$ suggested by the RDP. Furthermore rarefaction curves and richness indices were calculated using the relevant RDP pipeline modules as described elsewhere (Zhang et al., 2015).

\subsection{Data analysis}

The results of chemical parameters were visualized through Origin 9.0 (OrginLab, USA). A heat map of the top 10 genus in each sample based on reads $\log 2$ transformed was built using the HemI (http://hemi.biocuckoo.org/). Circos graph showing the evolution of bacterial community of each sample in different treatments at phylum level was produced by the Circos software (http://circos. $\mathrm{ca} /$ ). According to the relative content of each genus from the classification, a principal component analysis (PCA) was performed using Canoco 5.0 (Microcomputer Power, USA).

\section{Results and discussion}

\subsection{Effects of MW on anaerobic co-digestion of FW and SS}

As shown in Fig. 1A, all the biogas production in anaerobic codigestion of FW and SS with and without MW could be enhanced significantly in comparison with mono-FW and mono-SS. After 35 days of BMP tests, the cumulative methane production was calculated to be $70.72,142.70,296.75,311.26$ and $317.92 \mathrm{mLCH}_{4} /$ $\mathrm{gVS}_{\text {added }}$ for mono-FW, mono-SS, FW:SS (1:1), MW-FW:SS (1:1) and FW:MW-SS (1:1), respectively. FW mainly consisted of polysaccharides, while SS was mainly constituted by proteins (Table 1). The anaerobic degradability of poly-saccharides was much higher than proteins (Tommaso et al., 2003). This could be confirmed by the maximum daily methane production of mono-FW on day 1 , while mono-SS on days $1-8$. The least biogas production of mono-FW maybe due to the acidification as previously suggested (Kawai et al., 2014), while the cumulative methane yields of mono-SS were comparable to previous study in which the hydrolysis was considered as the rate-limited step for anaerobic digestion of SS (Liu et al., 2015). The advantage of anaerobic co-digestion of FW and SS has been generally confirmed (Duan et al., 2012). The daily methane production reached a peak on day 1 and decreased significantly like mono-FW, and the daily methane production in co-digestion of FW and SS increased gradually from day 4 and reached another peak on day 12 because the addition of SS served as a buffer. The methane yields of FW:SS (1:1) increased by 4.20 and 2.08 times in comparison with mono-FW and mono-SS, respectively, and the removals of TCOD, SCOD, TS and VS were also significantly enhanced after the co-digestion of FW and SS (Table 2).

It is important to highlight that both MW-FW and MW-SS could further enhance the methane production and TCOD removal (Table 2). The methane production further increased by $4.89 \%$ and $7.13 \%$ after MW-FW and MW-SS, respectively. It was assumed that the enhancement of co-digestion of FW and SS was higher through MW-SS compared to MW-FW. This was also confirmed by the highest peak value daily methane production of FW: MW-SS (1:1). It is easy to understand the effects of MW pretreat- ment of SS on the anaerobic co-digestion of FW and SS. The MW pretreatment to enhance anaerobic digestion of SS has been widely investigated (Eskicioglu et al., 2007a,b). The main fraction of SS consists of a polymeric network formed by extracellular polymeric substances (EPS) and microbial cells that are resistant to direct anaerobic degradation since cell walls and EPS present physical and chemical barriers, while MW pretreatment can achieve higher SS floc, cell destruction and release of EPS and intracellular materials into the soluble phase, and this increased biogas production. The increased SCOD after MW pretreatment could elucidate this assumption (Table 1).

Maillard compounds start to form at temperatures above $100{ }^{\circ} \mathrm{C}$ depending on the retention time, and the formation of more complex compounds, such as acrylamides and other vinylogous compounds, increases at higher temperatures like $180^{\circ} \mathrm{C}$ (Stadler et al., 2004), thus, low temperature MW pretreatment of FW was adopted in this study. MW-FW could also further increase the biogas production for the co-digestion of FW and SS. The enhancement of MW-FW to anaerobic digestion has also been confirmed previously, and the solubilization of FW could be increased (Table 1). However, the enhancement of co-digestion was slightly lower for the MW-FW, and this could be explained by higher enhancement of release of SCOD of MW-SS (ca. 2.0 times) in comparison with MW-FW (ca. 1.2 times). However, slightly lower TS and VS removal (Table 2) compared with the co-digestion of FW and SS without MW indicated the formation of complex compounds which were hard to be biodegraded after MW pretreatment.

\subsection{Optimized ratio of FW and SS for anaerobic co-digestion based on MW}

In order to determine the optimized ratio of FW and SS based on MW, BMP tests of five ratio of FW and SS (2:1, 3:2, 1:1, 2:3 and 1:2) (TS) with MW-FW or MW-SS were further conducted. The optimized ratio of FW and SS was always 3:2 (TS) for both MW-FW and MW-SS as revealed by Fig. $1 \mathrm{~B}$ and $\mathrm{C}$, and the detail methane production was listed in Table 2 . The maximum methane production was $316.24,338.44 \mathrm{mLCH}_{4} / \mathrm{gVS}_{\text {added }}$ for MW-FW, MW-SS at the optimized ratio of $3: 2$, respectively, and the methane production of MW-SS was $6.94 \%$ higher than that of MW-FW at the same ratio. These results further confirmed that the enhancement was higher for co-digestion of FW and SS through MW-SS instead of MW-FW.

The daily biogas production showed a similar profile at different treatments. It peaked on day 1 , and then decreased significantly, while increased on day 4 . The active phase for methane production ranged from day 5 to 16 . Polysaccharides removal rate was generally higher for the MW-FW compared to that of MW-SS, while proteins removal rate was much higher for the MW-SS in comparison with the MW-FW (Table 2). The final $\mathrm{NH}_{4}^{+}-\mathrm{N}$ was generally higher for MW-SS at the same ratio, which corresponded to higher proteins removal. TS and VS removals decreased along with the increasing addition of SS for both MW-FW and MW-SS (Table 2). This could be explained by the relative low TS and VS removal in comparison with FW. Initial and final $\mathrm{NH}_{4}^{+}-\mathrm{N}$ also showed the same pattern. Although TCOD removal decreased along with the increasing addition of SS for MW-FW, SCOD, polysaccharides and proteins removals were the highest at the ratio of 3:2 (MW-FW:SS). Previous studies have shown that the degradation of proteins is progressively retarded with increased concentrations of carbohydrates present (Tommaso et al., 2003). The highest methane production at this ratio could be due to the optimization of the degradation of polysaccharides and proteins. However, as for MW-SS, the highest methane production could be associated with the highest TCOD 

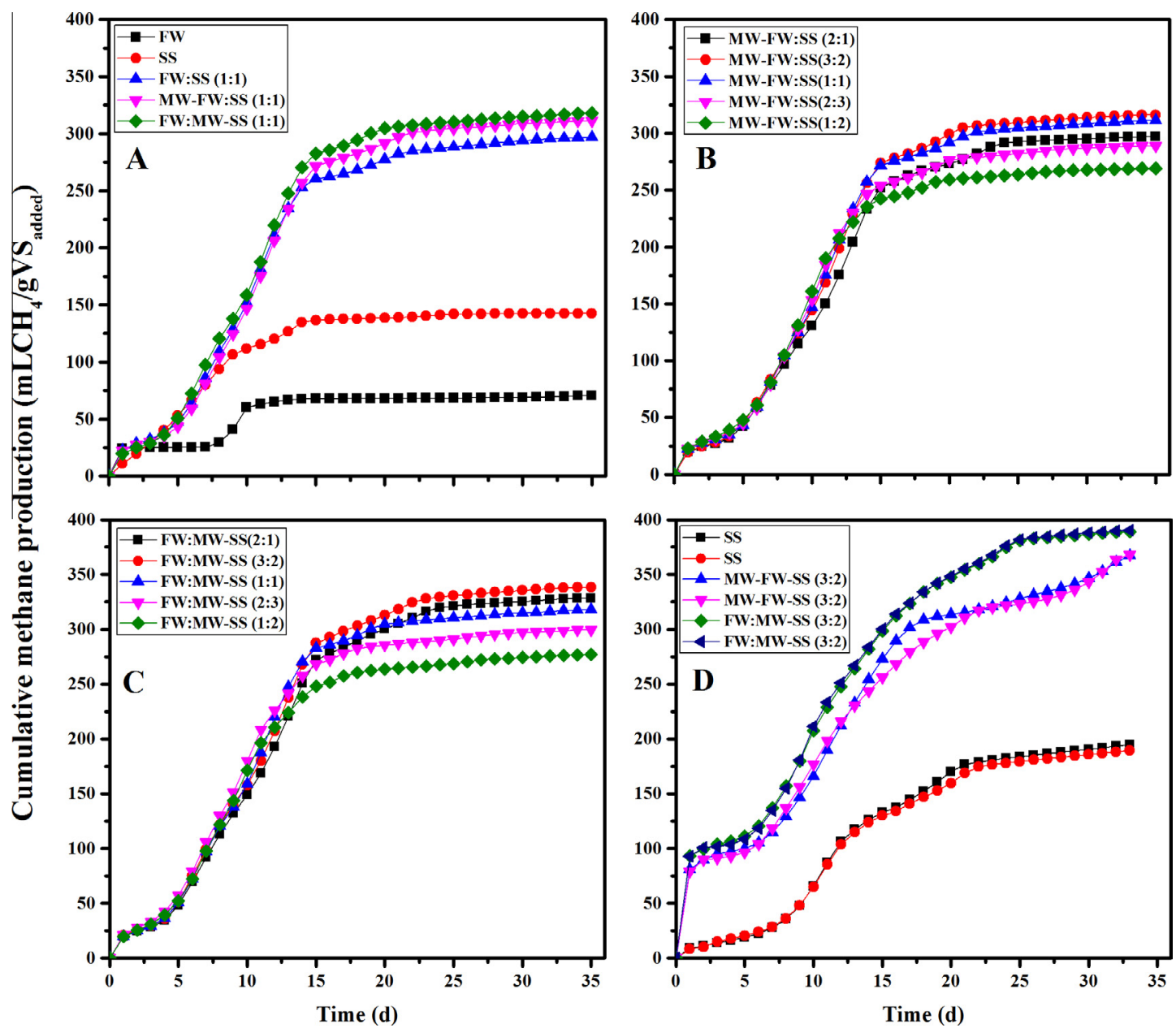

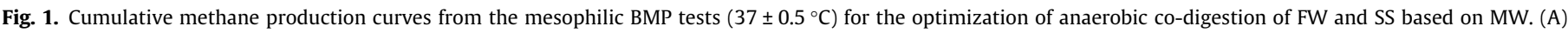

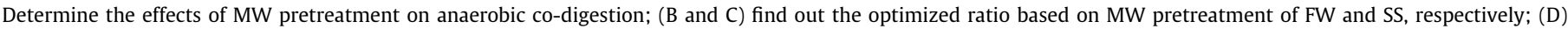
conducted in $1.8 \mathrm{~L}$ working volume bottles in duplicate for each treatment to explore the underlying mechanisms.

removal at the optimized ratio. The different mechanisms behind MW-FW and MW-SS needed to be further elucidated.

\subsection{Mechanisms of anaerobic co-digestion underlying the optimized ratio based on MW}

Further studies concerning the underlying mechanisms of anaerobic co-digestion of FW and SS at the optimized ratio based on MW were conducted to find out the different effects on anaerobic co-digestion between MW-FW and MW-SS. Previous studies have shown that MW pretreatment is more effective for sludge anaerobic digestion with high solids concentration (Eskicioglu et al., 2007a,b), thus, the TS was doubled to enhance the anaerobic co-digestion of FW and SS. BMP tests of mono-SS, MW-FW:SS (3:2) and FW:MW-SS (3:2) were further re-conducted in duplicate in a bigger scale to monitor the evolution of relative parameters. Mono-FW anaerobic digestion was not conducted due to its end of methane production untimely as suggested previously (Fig. 1A).

\subsubsection{Evolution of chemical parameters and VFAs}

Higher solids concentration indeed increased methane production (Table 2), and the methane production was 191.90, 367.62 and $389.64 \mathrm{mLCH}_{4} / \mathrm{gVS}_{\text {added }}$ for mono-SS, MW-FW:SS (3:2) and FW: MW-SS (3:2), respectively. Compared to mono-SS, co-digestion of FW and SS based on MW pretreatment increased by 1.92 and 2.03 times, respectively. Furthermore, MW-SS showed its advantage of enhancement of anaerobic co-digestion in comparison with MW-FW (Fig. 1D). The final methane production of MW-SS was $5.99 \%$ higher than that of MW-FW. Concerning the daily methane production, the similar pattern to previous BMP tests was developed, i.e., peak on day 1 , another peak on day 10 and active methane production on days 5-16. All the peaks were generally higher for MW-SS than that of MW-FW.

MW-SS showed lower $\mathrm{pH}$ and $\mathrm{NH}_{4}^{+}-\mathrm{N}$ concentrations throughout the tests, and there was a peak value of SCOD on day 5 for all the treatments. However, the peak value for MW-FW (34, $120 \mathrm{mg} / \mathrm{L})$ and $\mathrm{SS}(31,810 \mathrm{mg} / \mathrm{L})$ was much higher than that of mono-SS $(18,720 \mathrm{mg} / \mathrm{L})$. The increase of SCOD for mono-SS could be due to the release of refractory substances, and this was further confirmed by the increase of polysaccharides and proteins concentrations. MW-SS showed higher TCOD, SCOD, TS, VS and proteins removal but lower polysaccharides removal in comparison with MW-FW, and the significant reduction of polysaccharides could explain the peak daily methane production of co-digestion.

Changes of VFAs concentration and composition could be used to indicate the performance of anaerobic digestion (Wang et al., 2014). The accumulation of VFAs appeared on day 5 for all the treatments, and then it decreased gradually (Fig. 2). The average peak value of VFAs concentration was 9, 354.34, 23, 022.8 and $24,537.9 \mathrm{mg} / \mathrm{L}$ for mono-SS, MW-FW and MW-SS, respectively. The highest release of VFAs for MW-SS determined its highest methane production. VFAs are subsequently decreased as a result 

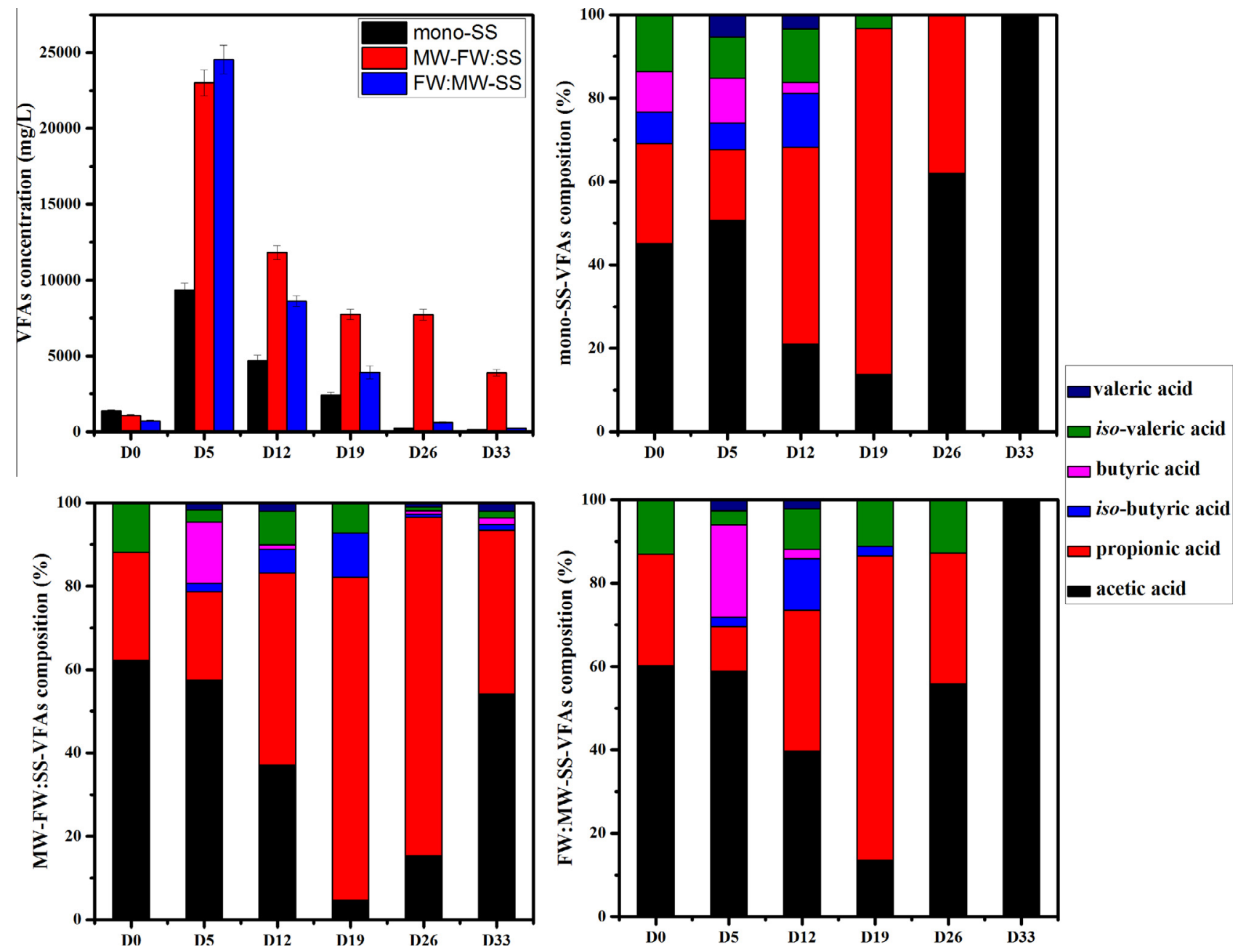

Fig. 2. Changes of VFAs concentrations and composition along with anaerobic digestion for different treatments.

of their uptake by the anaerobic microorganisms. The composition of VFAs changed significantly along with anaerobic digestion. Acetic acid was predominant on day $0-12$, and also peaked on day 5. As for MW-SS and mono-SS, acetic acid decreased along with anaerobic digestion, and little acetic acid left at the final (241.48 and $149.80 \mathrm{mg} / \mathrm{L}$, respectively). Although propionic acid was difficult to be utilized, there was no accumulation for MW-SS and mono-SS. Propionic acid, valeric acid, iso-valeric acid, butyric acid and iso-butyric acid all decreased to ND (not detected). However, it is quite different for MW-FW, propionic acid accumulated to $6262.96 \mathrm{mg} / \mathrm{L}$ on day 26 , while acetic acid decreased on day $12-19$, it rebounded to $2103.57 \mathrm{mg} / \mathrm{L}$ at the final. This indicated that higher propionic acid accumulation has led to inhibition of methanogenesis which further contributed to the accumulation of acetic acid. It was assumed that accumulation of propionic acid was the reason for the lower methane production of MW-FW in comparison with MW-SS.

\subsubsection{Evolution of bacterial community}

Detailed evolution of bacterial community was investigated to further find out underlying mechanisms of the difference between the effects of MW-FW and MW-SS on anaerobic co-digestion of FW and SS. After chimer analysis, a total of 499, 307 high quality reads for the 15 samples ranging from 32, 422 to 34,461 reads were generated for further analysis. The evolution of bacterial alpha indexes was shown in Supporting information. These high quality reads were assigned to different taxa levels using the RDP classifier. A total of 33 phylum were identified, and Firmicutes, Proteobacteria, Bacteroidetes and Actinobacteria were the predominant phylum in the bacterial community as shown in Fig. 3A. Firmicutes was enriched by 5.05, 6.45 and 4.61 times for mono-SS, MW-SS and MW-FW, respectively. The abundance of Firmicutes all increased significantly on day 5 . After that, Firmicutes changed little for mono-SS and MW-SS while the abundance increased much further on day 12 for MW-FW and changed little to the end. The evolution of Proteobacteria was quite different for different treatments especially for mono-SS. The abundance of Proteobacteria decreased significantly for MW-SS and MW-FW in comparison with mono-SS, and it was the maximum contributor to the difference of mono-SS and co-digestion. The phylum Actinobacteria showed similar evolution to Proteobacteria. As for Bacteroidetes, the abundance decreased a little for mono-SS while increased for MW-SS and MW-FW. There was a common phenomenon for these four dominant phylum that the maximum or minimum abundance, that is, greatest changes, all appeared on day 5, which indicated that recombination of bacterial community occurred at acidification phase corresponding to VFAs accumulation.

The top 10 abundant genus in each sample (a total of 61 genus) were selected for a detailed understanding of the evolution of microbial community structure as suggested previously (Zhang et al., 2015). Heat map shown in Fig. 3B indicated the evolution of each genus selected in different treatments. PCA analysis based on these genus showed the changes of microbial community, which indicated the significant difference between mono-SS and 

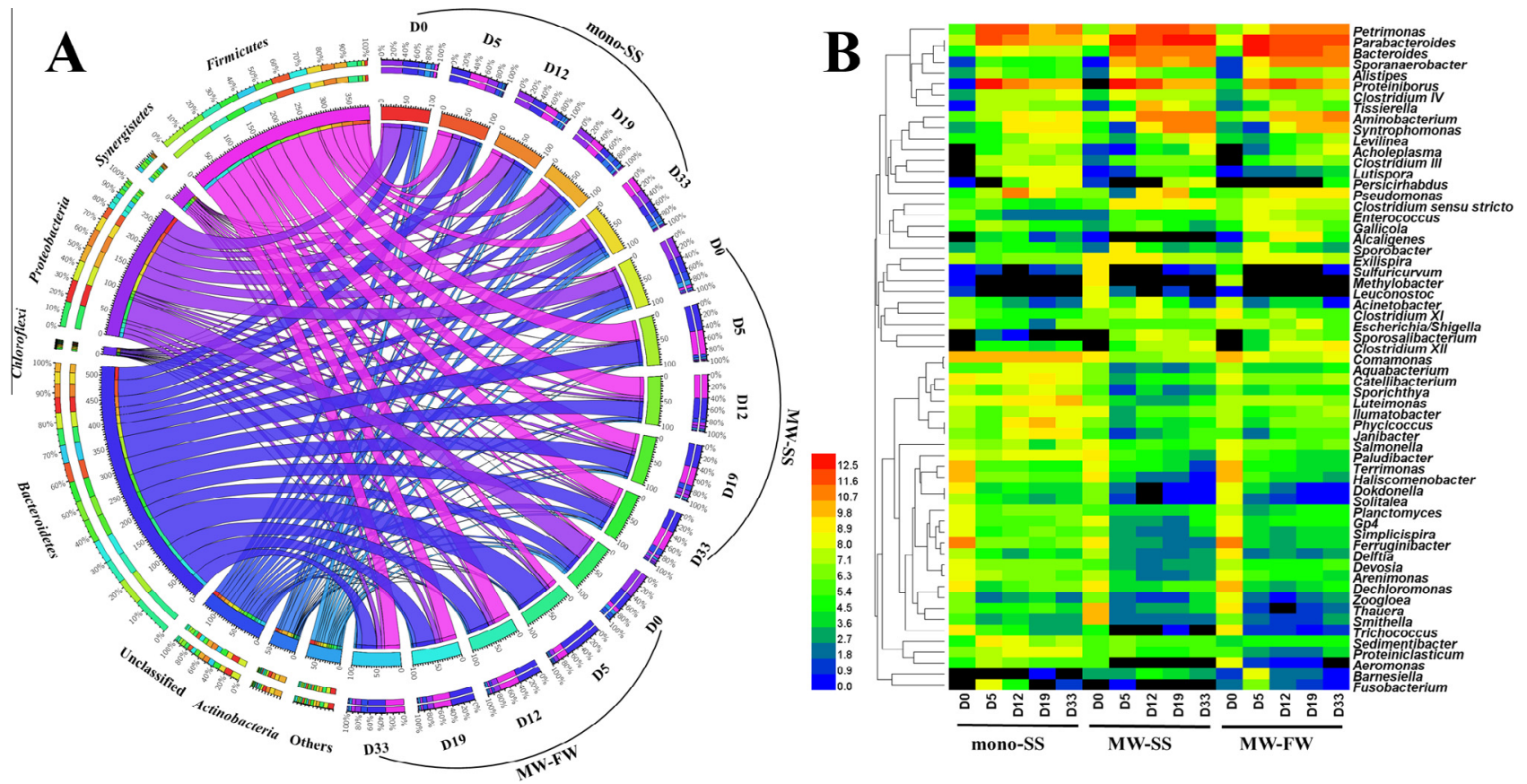

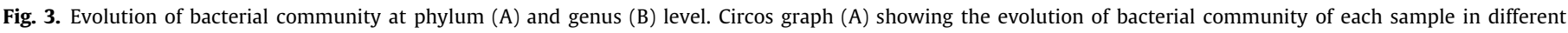

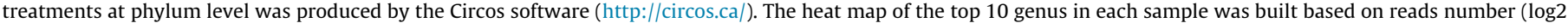
transformed).

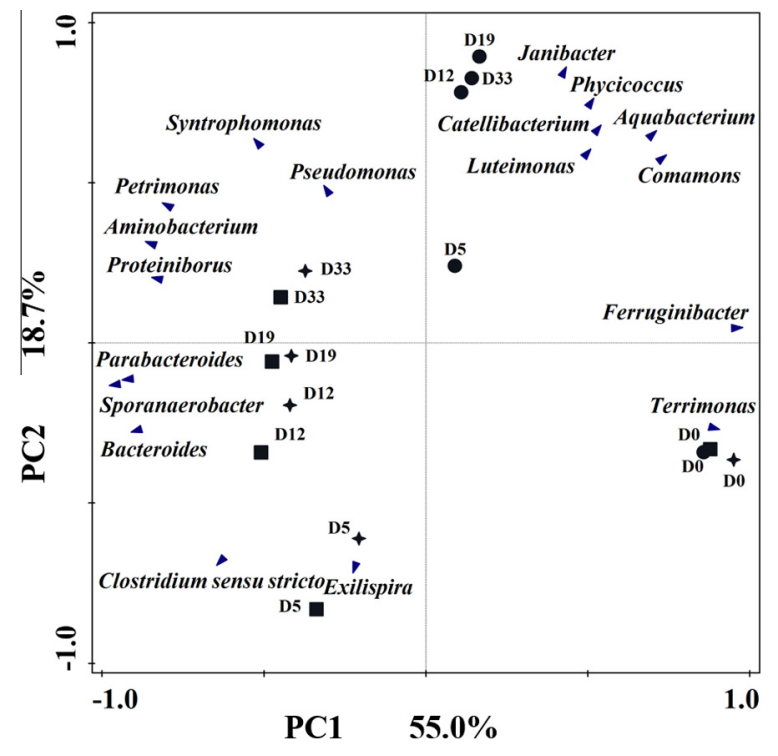

Fig. 4. Principal component analysis (PCA) based on the top 10 abundant genus Solid circle, square and star indicated samples from mono-SS, MW-SS and MW-FW, respectively, while solid triangle represented the key genus which contribute largely to bacterial different samples.

co-digestion of FW and SS based on MW (Fig. 4). Changes of bacterial community could be divided into two phases based on PCA analysis: days $0-5$, acidification phase corresponding to accumulation of VFAs and days 5-33, active methane production phase corresponding to consumption of VFAs (Fig. 2). The greatest changes happened at acidification phase for all the treatments, and so the difference between mono-SS and co-digestion based on MW-FW and MW-SS did. Proteiniborus and Parabacteroides were the dominant genus for the VFAs production in all the tests, while Petrimonas was only dominant at the acidification phase for monoSS. Correspondingly, Bacteroides which changed little for mono-SS increased significantly and dominated on day 5 for co-digestion. After acidification phase, most of the dominant genus decreased along with anaerobic digestion.

Proteiniborus belonging to phylum Bacteroidetes is a kind of anaerobic, mesophilic and protein-specific utilizing bacteria, and the fermentation products mainly include ethanol, acetic acid, hydrogen, carbon dioxide and a trace amount of propionic acid (Niu et al., 2008). It is easy to understand its dominance for anaerobic digestion of SS which was mainly composed of proteins, and it is the first abundant genus for mono-SS (18.81\%) and MW-SS (23.64\%) anaerobic digestion. Meanwhile, its abundance was the lowest $(5.52 \%)$ for MW-FW, and this was because MW-SS could help release proteins in bacteria. Parabacteroides also belonging to Bacteroidetes is saccharolytic and produces acetate and succinate as its primary fermentation end products (Tan et al., 2012). That is to say that members of Parabacteroides are responsible for the degradation of polysaccharides in all the treatments. Thus, its abundance was higher in co-digestion due to the addition of FW whose main components were polysaccharides. Petrimonas is described as a mesophilic, strictly anaerobic and fermentative bacterium, and acetic acid was the major end products (Grabowski et al., 2005). It contributed more to VFAs accumulation of monoSS than that of MW-SS and MW-FW, because its abundance increased significantly on day 12 for MW-SS (14.59\%) and MWFW (9.26\%), 7 days later than mono-SS. It is interesting to notice that Bacteroides only dominated for the co-digestion. Members of genus Bacteroides were isolated from various environments, and most of them produce acetic acid, propionate acid, formate and succinic acid as the major end-products of fermentation (Hatamoto et al., 2014). Especially for MW-FW, its abundance accounted for $32.66 \%$, which indicated that it contributed most to the VFAs accumulation of MW-FW.

Besides, other microbes only increased during anaerobic digestion of mono-SS were revealed by PCA analysis (Fig. 4) such as Janibacter, Comamonas, Luteimonas. These genus only increased in mono-SS, while decreased or changed little for the co-digestion based on MW pretreatment. But their specific function for anaerobic 


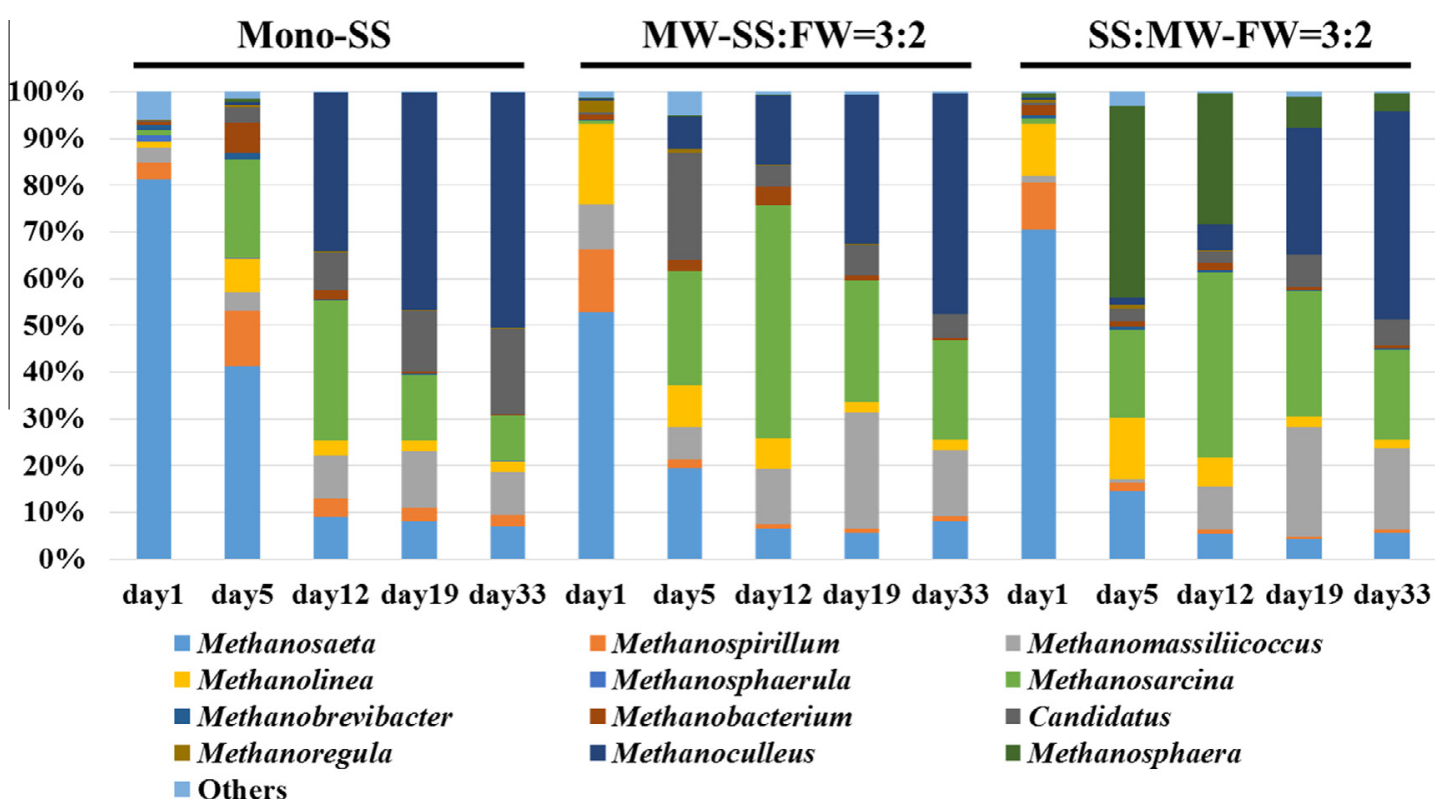

Fig. 5. Evolution of archaeal community in different treatments at genus level.

digestion of mono-SS needed further research. Some specific protein-degrading bacteria like Aminobacterium increased after day 5 for MW-SS and MW-FW, because proteins were not easily available compared to polysaccharides. It is a kind of protein-degrading bacteria, and acetate, succinate and propionate were the predominant fermentation products. Meanwhile Sporanaerobacter could also degrade proteins in addition to sugars, and its abundance only increased for co-digestion. Syntrophomonas all increased at the end of anaerobic digestion, and it is a kind of butyrate oxidizing syntrophic bacteria with methanogenesis. Its significant increase of abundance was comparable to the decline of butyrate (Fig. 2).

\subsubsection{Evolution of archaeal community}

Although primers of 16S rRNA V4 targeting both bacterial and archaeal community were used, lower abundance of archaeal community (ranging $0.29-1.01 \%$ shown in bacterial community analysis at phylum) made it impossible to analyze the composition of archaea in detail. Thus, a total of 479,911 high quality reads ranging from 30, 124 to 34, 910 were acquired for detailed archaeal community analysis. According to RDP classifier, most of these reads were assigned to methanogenic archaea. Compared to bacterial community, the diversity of archaeal community was very simple, while significant changes happened along with anaerobic digestion as shown in Fig. 5. The acetoclastic Methanosaeta dominated on day 0 accounting for $81.23 \%, 52.9 \%$ and $70.53 \%$ for mono-SS, MW-SS and MW-FW, respectively, while its abundance decreased significantly along with anaerobic digestion and the final abundance was $6.98 \%, 8.14 \%$, and $5.7 \%$, respectively. Meanwhile, another acetoclastic methanogen, Methanosarcina, increased significantly at the active methane production phase (day 12) for all the treatments. The maximum abundance at this phase was $30.09 \%, 49.92 \%$ and $39.78 \%$, that is, increased by $28.66,97.88$ and 37.53 times for mono-SS, MW-SS and MW-FW, respectively. After that, the abundance of Methanosarcina decreased to $9.6 \%, 21.2 \%$ and $19.34 \%$, respectively. The decline of Methanosaeta and increase of Methanosarcina indicated that there existed a competitive growth between these two acetoclastic methanogens. Genus Methanosarcina has been reported to have high growth rates (i.e., doubling rates on the order of 1.0-1.2 d) and are more tolerant to sudden changes in $\mathrm{pH}$ of around $0.8-1.0$ units, as compared to the other methanogens, which have doubling times of a minimum of 4-6 d and tend to be affected by a pH shock of 0.5 units or even less (Cho et al., 2013). In addition, Methanosarcina are able to use both the acetoclastic and the hydrogenotrophic methanogenesis pathways, making them more tolerant to specific inhibitors of the acetoclastic pathway compared to Methanosaeta. Accumulation of VFAs on day 5 (Fig. 2) made it predominant advantage during competing with Methanosaeta. However, hydrogenotrophic methanogens of Methanoculleus became dominant at the end of anaerobic digestion due to exhaust of acetic acid. Methanoculleus-like archaea can use $\mathrm{CO}_{2}$ or formate as carbon source and $\mathrm{H}_{2}$ as electron donor and not from acetate to produce methane. The final abundance of Methanoculleus was $50.42 \%, 47.34 \%$ and $44.64 \%$, while its initial abundance was ND, $0.57 \%$ and $0.49 \%$ for mono-SS, MW-SS and MW-FW, respectively.

The most interesting phenomenon appeared during anaerobic co-digestion of MW-FW, that is, genus Methanosphaera dominated on day 5 (from $0.84 \%$ to $40.76 \%$ ), and its evolution was quite special for MW-FW and subsequently decreased gradually along with anaerobic digestion to the end (3.83\%) (Fig. 5). Members of genus Methanosphaera have one of the most restricted energy metabolisms of all methanogens because it can neither oxidize methanol to $\mathrm{CO}_{2}$, nor reduce $\mathrm{CO}_{2}$ to methane (Fricke et al., 2006). Instead, it is dependent on acetate as a main carbon source for growth and uses $\mathrm{H}_{2}$ to reduce methanol to produce methane (Fricke et al., 2006). Previous study have demonstrated that high levels of methanol and acetate would provide an ideal environment for Methanosphaera to thrive (Facey et al., 2012), and this indicated that MW pretreatment of FW may produce amounts of methanol.

\section{Conclusions}

Anaerobic co-digestion of FW and SS was generally considered effective for methane production. The effects of MW for the co-digestion have never been investigated. Results showed that the optimized ratio of FW and SS based on MW pretreatment was 3:2 (TS), no matter FW or SS was MW pretreatment, and MW-FW was less efficient for enhancing the methane production than MW-SS due to the accumulation of propionic acid. Co-digestion of FW and SS based on MW changed the composition of bacterial and archaeal community significantly, and the 
evolution of them was studied in detail through high-throughput sequencing method.

\section{Acknowledgements}

This work is supported by Major Science and Technology Program for Water Pollution Control and Treatment of China (2012ZX07202-005, 2015ZX07203-007) and the National Natural Science Foundation of China (21377151).

\section{Appendix A. Supplementary data}

Supplementary data associated with this article can be found, in the online version, at http://dx.doi.org/10.1016/j.biortech.2015.10. 037.

\section{References}

APHA, AWWA, WEF, 2005. Standard Methods for the Examination of Water and Wastewater, twenty-first ed. American Public Health Association, Washington, DC.

Baier, U., Schmidheiny, P., 1997. Enhanced anaerobic degradation of mechanically disintegrated sludge. Water Sci. Technol. 36, 137-143.

Caporaso, J.G., Lauber, C.L., Walters, W.A., Berg-lyons, D., Lozupone, C.A., Turnbaugh, P.J., Fierer, N., Knight, R., 2010. Global patterns of 16S rRNA diversity at a depth of millions of sequences per sample. PNAS 108, 1516-1522.

Cho, S.K., Im, W.T., Kim, D.H., Kim, M.H., Shin, H.S., Oh, S.E., 2013. Dry anaerobic digestion of food waste under mesophilic conditions: performance and methanogenic community analysis. Bioresour. Technol. 131, 210-217.

Coelho, N.M.G., Droste, R.L., Kennedy, K.J., 2011. Evaluation of continuous mesophilic, thermophilic and temperature phased anaerobic digestion of microwaved activated sludge. Water Res. 45, 2822-2834.

Duan, N., Dong, B., Wu, B., Dai, X., 2012. High-solid anaerobic digestion of sewage sludge under mesophilic conditions: feasibility study. Bioresour. Technol. 104, $150-156$.

Edgar, R.C., Haas, B.J., Clemente, J.C., Quince, C., Knight, R., 2011. UCHIME improves sensitivity and speed of chimera detection. Bioinformatics 27, 2194-2200.

Eskicioglu, C., Droste, R.L., Kennedy, K.J., 2007a. Performance of anaerobic waste activated sludge digesters after microwave pretreatment. Water Environ. Res. 79, 2265-2273.

Eskicioglu, C., Terzian, N., Kennedy, K.J., Droste, R.L., Hamoda, M., 2007b. Athermal microwave effects for enhancing digestibility of waste activated sludge. Water Res. 41, 2457-2466.

Facey, H.V., Northwood, K.S., Wright, A.-D.G., 2012. Molecular diversity of methanogens in fecal samples from captive Sumatran orangutans (Pongo abelii). Am. J. Primatol. 74, 408-413.

Fricke, W.F., Liesegang, H., Hedderich, R., Gottschalk, G., Thauer, R.K., 2006. The genome sequence of Methanosphaera stadtmanae reveals why this human intestinal archaeon is restricted to methanol and $\mathrm{H}_{2}$ for methane formation and ATP synthesis. J. Bacteriol. 188, 642-658.

Gantner, S., Andersson, A.F., Alonso-Sáez, L., Bertilsson, S., 2011. Novel primers for $16 \mathrm{~S}$ rRNA-based archaeal community analyses in environmental samples. J. Microbiol. Methods 84, 12-18.

Grabowski, A., Tindall, B.J., Bardin, V., Blanchet, D., Jeanthon, C., 2005. Petrimonas sulfuriphila gen. nov., sp. nov., a mesophilic fermentative bacterium isolated from a biodegraded oil reservoir. Int. J. Syst. Evol. Microbiol. 55, 1113-1121.
Hatamoto, M., Kaneshige, M., Nakamura, A., Yamaguchi, T., 2014. Bacteroides luti sp. nov., an anaerobic, cellulolytic and xylanolytic bacterium isolated from methanogenic sludge. Int. J. Syst. Evol. Microbiol. 64, 1770-1774.

Kawai, M., Nagao, N., Tajima, N., Niwa, C., Matsuyama, T., Toda, T., 2014. The effect of the labile organic fraction in food waste and the substrate/inoculum ratio on anaerobic digestion for a reliable methane yield. Bioresour. Technol. 157, 174180.

Lee, M., Hidaka, T., Hagiwara, W., Tsuno, H., 2009. Comparative performance and microbial diversity of hyperthermophilic and thermophilic co-digestion of kitchen garbage and excess sludge. Bioresour. Technol. 100, 578-585.

Liu, J., Tong, J., Wei, Y., Wang, Y., 2015. Microwave and its combined processes: an effective way for enhancing anaerobic digestion and dewaterability of sewage sludge? J. Water Reuse Desalin. 2, 1-21.

Magoč, T., Salzberg, S.L., 2011. FLASH: fast length adjustment of short reads to improve genome assemblies. Bioinformatics 27, 2957-2963.

Marin, J., Kennedy, K.J., Eskicioglu, C., 2010. Effect of microwave irradiation on anaerobic degradability of model kitchen waste. Waste Manage. 30, 1772-1779.

Nges, I.A., Liu, J., 2009. Effects of anaerobic pre-treatment on the degradation of dewatered-sewage sludge. Renew. Energy 34, 1795-1800.

Niu, L., Song, L., Dong, X., 2008. Proteiniborus ethanoligenes gen. nov., sp. nov., an anaerobic protein-utilizing bacterium. Int. J. Syst. Evol. Microbiol. 58, 12-16.

Schmieder, R., Edwards, R., 2011. Quality control and preprocessing of metagenomic datasets. Bioinformatics 27, 863-864.

Shahriari, H., Warith, M., Hamoda, M., Kennedy, K., 2013. Evaluation of single vs. staged mesophilic anaerobic digestion of kitchen waste with and without microwave pretreatment. J. Environ. Manage. 125, 74-84.

Stadler, R.H., Robert, F., Riediker, S., Varga, N., Davidek, T., Devaud, S., Goldmann, T., Hau, J., Blank, I., 2004. In-depth mechanistic study on the formation of acrylamide and other vinylogous compounds by the Maillard reaction. J. Agric. Food Chem. 52, 5550-5558.

Takai, K., Horikoshi, K., 2000. Rapid detection and quantification of members of the archaeal community by quantitative PCR using fluorogenic probes. Appl. Environ. Microbiol. 66, 5066-5072.

Tan, H.Q., Li, T.T., Zhu, C., Zhang, X.Q., Wu, M., Zhu, X.F., 2012. Parabacteroides chartae sp. nov., an obligately anaerobic species from wastewater of a paper mill. Int. J. Syst. Evol. Microbiol. 62, 2613-2617.

Tommaso, G., Ribeiro, R., Varesche, M.B., Zaiat, M., Foresti, E., 2003. Influence of multiple substrates on anaerobic protein degradation in a packed-bed bioreactor. Water Sci. Technol. 48, 23-31.

Toreci, I., Kennedy, K.J., Droste, R.L., 2009. Evaluation of continuous mesophilic anaerobic sludge digestion after high temperature microwave pretreatment. Water Res. 43, 1273-1284.

Wang, K., Yin, J., Shen, D., Li, N., 2014. Anaerobic digestion of food waste for volatile fatty acids (VFAs) production with different types of inoculum: effect of $\mathrm{pH}$. Bioresour. Technol. 161, 395-401.

Wei, Y., Van Houten, R.T., Borger, A.R., Eikelboom, D.H., Fan, Y., 2003. Minimization of excess sludge production for biological wastewater treatment. Water Res. 37, 4453-4467.

Yang, G., Zhang, G., Wang, H., 2015. Current state of sludge production, management, treatment and disposal in China. Water Res. 78, 60-73.

Yong, Z., Dong, Y., Zhang, X., Tan, T., 2015. Anaerobic co-digestion of food waste and straw for biogas production. Renew. Energy 78, 527-530.

Zhang, R., El-Mashad, H.M., Hartman, K., Wang, F., Liu, G., Choate, C., Gamble, P., 2007. Characterization of food waste as feedstock for anaerobic digestion. Bioresour. Technol. 98, 929-935.

Zhang, J., Cai, X., Qi, L., Shao, C., Lin, Y., Zhang, J., Zhang, Y., Shen, P., Wei, Y., 2015. Effects of aeration strategy on the evolution of dissolved organic matter (DOM) and microbial community structure during sludge bio-drying. Appl. Microbiol. Biotechnol. 99, 7321-7331.

Zhu, Z., Hsueh, M.K., He, Q., 2011. Enhancing biomethanation of municipal waste sludge with grease trap waste as a co-substrate. Renew. Energy 36, 1802-1807. 